\title{
Relationship between Chief Complaints and Severity of Periodontitis in Patients Seeking Periodontal Therapy: A Retrospective Study
}

\author{
Subasree Soundarajan ${ }^{1}$, Sankari Malaippan ${ }^{2}$, Priya L Gajendran ${ }^{3}$
}

\begin{abstract}
Aim: The aim of the study was to assess the relationship between chief complaints (CCs) and severity of periodontitis in patients seeking periodontal treatment at Saveetha Institute of Medical and Technical Sciences (SIMATS), Chennai.

Materials and methods: This retrospective study was carried out by analyzing the patient records from June 2019 to March 2020 . Two hundred and forty-four patients who reported to the Department of Periodontics, seeking periodontal therapy in this period were included in the study. Parameters assessed were (a) demographic details — patient name, patient identity number, age, and sex; (b) periodontal parameters — periodontal probing depth (PDD), loss of attachment (LOA), bleeding on probing (BOP), severity of periodontitis (mild, moderate, and severe); (c) CCs and type of CCs (chronic symptomatic, acute symptomatic, and asymptomatic). Chi-square test was performed to assess the relationship between type of CC and severity of periodontitis using SPSS version 23.0 (Statistical Package for the Social Sciences).

Results: The overall distribution of the CCs shows that most common CC was bleeding gums (22.95\%), second most common CC was missing teeth (17.62\%), and third most common CC was tooth pain (15.68\%), followed by mobile teeth (14.75\%), swollen gums (9.02\%), teeth moved position $(6.56 \%)$, sensitivity $(5.33 \%)$, pain on chewing $(2.05 \%)$, bad breath $(2.05 \%)$, pus discharge $(1.64 \%)$, pain in gums $(0.82 \%)$, need gum treatment $(0.82 \%)$, and cleaning $(0.41 \%)$. Chi-square test shows that there is a significant relationship between type of CC and severity of periodontitis with a $p$ value of 0.015 .

Conclusion: The most common CC reported was bleeding gums. But further longitudinal studies with larger sample size are needed to be performed.

Clinical significance: Patient's CC is considered as a vital component for providing competent and good quality healthcare. The severity of the disease is said to have an influence on the CCs reported by the patients.

Keywords: Gingivitis, Periodontal abscess, Periodontal diseases, Periodontitis.

World Journal of Dentistry (2020): 10.5005/jp-journals-10015-1760
\end{abstract}

\section{INTRODUCTION}

Periodontitis is a chronic inflammatory disease, characterized by destruction of the supporting structure of the teeth. Periodontal disease is multifactorial and is influenced by several risk factors. The role of gram-negative anaerobes in periodontal destruction is well known. Lately, the viruses have been recognized to play a major role in the pathogenesis of chronic periodontitis (CP). Among the viruses, cytomegalovirus (CMV) and Epstein-Barr virus (EBV) are of most importance. ${ }^{1}$ Chronic periodontitis is a host-mediated inflammatory disease which is characterized by elevated levels of various cytokines and inflammatory mediators. Tumor necrosis factor (TNF) is one such proinflammatory cytokine that causes periodontal tissue destruction. ${ }^{2}$ Interleukins like IL-1, $-6,-8$, and -12 have been previously proved to cause definitive periodontal destruction. Recently, interleukin-21 (IL-21) has obtained paramount importance in causing amplified tissue inflammation, damage, and destruction. ${ }^{3}$ Expression of peptides like endothelin-1, which is a potent vasoconstrictor, can increase the expression of cytokines like interleukin-1 $\beta$ (IL-1 $\beta$ ), tumor necrosis factor- $\alpha$ (TNF- $\alpha$ ), and IL- $6 .{ }^{4,5}$ Recent literature has established that periodontitis could be a probable risk factor for cardiovascular diseases, such as atherosclerosis, stroke, myocardial infarction, diabetes, and respiratory disorders. Periodontitis and chronic obstructive pulmonary disease (COPD) share a common risk factor profile. They manifest as chronic inflammation with underlying connective tissue destruction. ${ }^{6}$

\begin{abstract}
${ }^{1-3}$ Department of Periodontics, Saveetha Dental College and Hospitals, Saveetha Institute of Medical and Technical Sciences, Saveetha University, Chennai, Tamil Nadu, India

Corresponding Author: Sankari Malaippan, Department of Periodontics, Saveetha Dental College and Hospitals, Saveetha Institute of Medical and Technical Sciences, Saveetha University, Chennai, Tamil Nadu, India, Phone: +91 9840285905, e-mail: sankari@ saveetha.com
\end{abstract}

How to cite this article: Soundarajan S, Malaippan S, Gajendran PL. Relationship between Chief Complaints and Severity of Periodontitis in Patients Seeking Periodontal Therapy: A Retrospective Study. World J Dent 2020;11(5):396-401.

Source of support: Nil

Conflict of interest: None

The diagnosis of active phases of periodontal disease is a challenge for the clinicians. Over the last few years, several advanced radiographic techniques have been developed, such as cone beam computed tomography, which help in better treatment planning and hence the treatment of the disease. ${ }^{7}$ The early diagnosis and treatment planning in periodontitis is of huge importance, because it eventually leads to tooth loss and prosthetic replacement of these sites becomes difficult. Dental implants have become a crucial part of prosthetic rehabilitation in periodontitis over the last few 
years. ${ }^{8}$ Initial treatment of periodontal disease is performed by non-surgical periodontal therapy, which includes scaling, root planning, systemic antimicrobials, and plaque control methods. Various antimicrobials and chemotherapeutic agents, such as chlorhexidine, triclosan, and cetylpyridinium chloride, have been used in the management of periodontal diseases. Due to its multifactorial etiology and complex disease process, the treatment of periodontitis is still a difficult task to dentists. Hence, herbal remedies, such as triphala mouthwash and Hiora mouthwash, have been used to achieve antimicrobial, antioxidant, and antiinflammatory effects. ${ }^{9,10}$ When periodontitis is left untreated, it might lead to loss of teeth, function, and esthetics. ${ }^{11}$ One of the most common esthetic concerns associated with the periodontal tissue is gingival recession. ${ }^{12}$ Deep periodontal defects can be managed by regenerative procedures. For complete periodontal regeneration, delivery of growth factors are considered as an adjunct to bone grafts. ${ }^{13,14}$ In recent days, stem cells have been used for regenerative therapy. Dental tissues, such as the periodontal ligament (PDL), the dental pulp, and the tooth follicle, have been recognized as readily available sources of adult stem cells. ${ }^{15}$

Compared to the Western countries, the level of patient compliance and number of referrals for periodontal therapy in India are relatively less. Community-based screening shows high prevalence of periodontal disease in India. ${ }^{16}$ The concept of referral is unclear and still developing among Indian dentists. Apart from referral, factors like patient motivation and compliance may influence their decision on periodontal treatment. In clinical practice, the most crucial step in managing patients is defining the objectives of treatment protocol. This is dependent on the basis of the patient's requirement. ${ }^{17}$ The chief complaints (CCs) and reasons for seeking periodontal care are principal sources of information for the dentists. Periodontal diseases are usually inactive in nature, presenting with chronic or asymptomatic CCs. ${ }^{18,19}$ Patients' CCs correspond to their immediate need and their attitude toward periodontal treatment. Compared to other presenting symptoms, focusing on the CC can aid in early diagnosis and treatment of the disease.

Clinical objectives of dental practices rely simply on specific and clear CCs. ${ }^{20}$ A CC can be defined as a subjective statement given by the patient that explains the most significant symptoms that caused him or her to seek treatment. It is usually recorded in the patient's own words. Multiple complaints are recorded in a chronological order to assess the patient's perception of their problem. ${ }^{21}$ The urgency of the patient to seek dental treatment depends on the severity of their complaints. ${ }^{22}$ According to the American Dental Association, a patient's CC is considered as a vital component for providing competent and good quality healthcare. ${ }^{23}$ Recording CCs help in maintaining comprehensive information about patient care. The CC is the initial step in dental history taking and is a brief statement explaining the symptom and condition that demands a dental visit. The patient's initial comments aid in determining a differential diagnosis. Recording and identification of CCs are also considered the foundation for developing a rational treatment plan. ${ }^{24}$ This study was intended to assess the most common CCs of the patients seeking periodontal therapy and evaluate the relationship between CCs and severity of periodontitis at Saveetha Institute of Medical and Technical Sciences (SIMATS).

\section{Materials and Methods}

\section{Study Design}

This retrospective study was carried out by analyzing the patient records from June 2019 to March 2020. The study design was reviewed and approved by the Ethical Committee of SIMATS (Ethical approval number. SDC/SIHEC/2020/DIASDATA/0619-0320).

\section{Study Population}

Data from 244 patients who were diagnosed with CP and referred to the Department of Periodontics, SIMATS, were included in the study. Data were derived from the hospital's digital record management system called dental information archiving software. Patients with aggressive periodontitis or any systemic diseases that may influence the periodontium like diabetes, pregnancy, immunological disorders, and smokers were excluded from the study. Case sheets with incomplete data were excluded from the study.

\section{Parameters Assessed}

- Demographic details-Patient name, patient identity number, age, and sex.

- Periodontal parameters-Periodontal probing depth (PDD), loss of attachment (LOA), and bleeding on probing (BOP).

- Severity of periodontitis_-25

- Mild periodontitis-LOA $=<3 \mathrm{~mm}$.

- Moderate periodontitis-LOA $=3-4 \mathrm{~mm}$ at $\geq 30 \%$ sites.

- Severe periodontitis-LOA $\geq 5 \mathrm{~mm}$ at $\geq 30 \%$ of sites.

- Chief complaints and type of CCs (chronic symptomatic, acute symptomatic, and asymptomatic).

\section{Statistical Analysis}

All descriptive data were analyzed using frequency distributions; Chi-square test was performed to assess the relationship between type of CC and severity of periodontitis. Statistical significance was set at $p<0.05$. Analyses were conducted with a statistical software SPSS version 23.0 (Statistical Package for the Social Sciences).

\section{Results}

A total of two hundred and forty-four patients who reported to the Department of Periodontics, seeking periodontal therapy, were included in the study. Characteristics of the study population are described in Table 1. The mean age of the study sample was 40.42 ( \pm 11.38 ). Females constituted about $58.06 \%$, whereas males constituted $41.4 \%$ of the study sample. Mean probing depth was $5.24( \pm 1.30)$, mean LOA was $4.60( \pm 1.45)$, and mean BOP score was $0.98( \pm 0.25)$.

The overall distribution of the CCs shows that most common CC was bleeding gums (22.95\%), second most common CC was missing teeth (17.62\%), third most common CC was tooth pain $(15.68 \%)$, followed by mobile teeth (14.75\%), swollen gums $(9.02 \%)$, teeth moved position (6.56\%), sensitivity $(5.33 \%)$, pain on chewing $(2.05 \%)$, bad breath $(2.05 \%)$, pus discharge $(1.64 \%)$, pain in gums $(0.82 \%)$, need gum treatment (0.82\%), and cleaning (0.41\%) (Fig. 1). About $65 \%$ of the study population were diagnosed with severe periodontitis, $17.21 \%$ were diagnosed with moderate periodontitis, and $18.03 \%$ had mild periodontitis (Fig. 2). 
Table 1: Descriptive analysis of the study population

\begin{tabular}{lll}
\hline Gender & Number of patients & Percentage \\
\hline Female & 143 & 58.60 \\
Male & 101 & 41.4 \\
\hline Prevalence of & & \\
periodontitis & Number of patients & Percentage \\
\hline Mild & 44 & 18.03 \\
Moderate & 42 & 17.21 \\
Severe & 158 & 64.76 \\
\hline & Mean & Standard deviation \\
\hline Age & 40.82 & 11.38 \\
PPD (mm) & 5.24 & 1.30 \\
CAL (mm) & 4.60 & 1.45 \\
BOP score & 0.98 & 0.25 \\
\hline
\end{tabular}

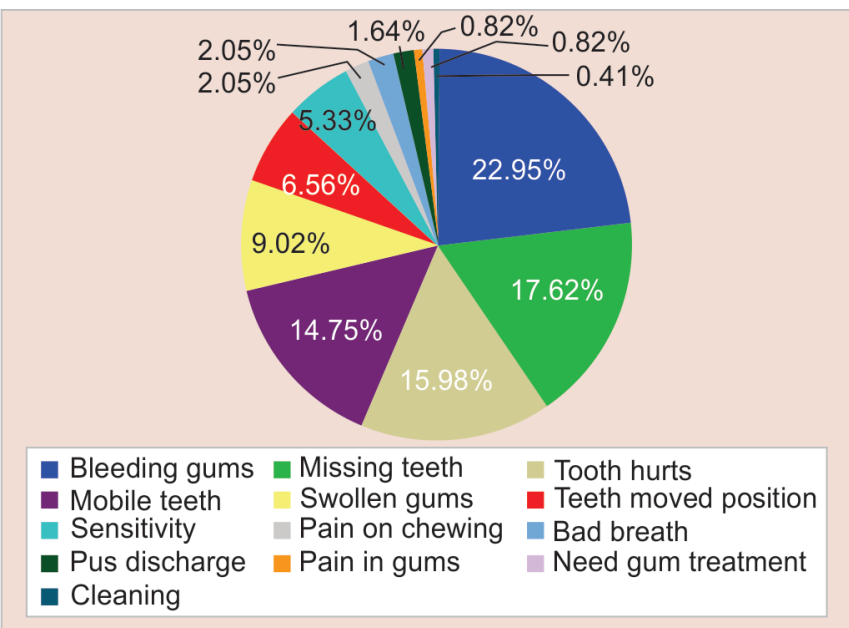

Fig. 1: Pie chart representing distribution of chief complaints. Chronic symptomatic CCs include bad breath, bleeding gums, mobile teeth, pain in gums, pain on chewing, pus discharge, sensitivity, swollen gums, and moved position; acute symptomatic CCs include toothache; asymptomatic CCs include cleaning, missing teeth, and need gum treatment. The most common chief complaint was bleeding gums $(22.95 \%)$, second most common chief complaint was missing teeth $(17.62 \%)$, and third most common chief complaint was tooth pain (15.98\%)

\section{Relationship between CC and Severity of Periodontitis (Fig. 3)}

Among the patients who reported with chronic symptomatic CCs, $7.79 \%$ of the patients were diagnosed with mild periodontitis, $12.7 \%$ of the patients were diagnosed with moderate periodontitis, and $44.67 \%$ of the patients were diagnosed with severe periodontitis. Among those who reported with acute symptomatic CCs, $4.51 \%$ of the patients were diagnosed with mild periodontitis, $1.64 \%$ of the patients were diagnosed with moderate periodontitis, and $9.84 \%$ of the patients were diagnosed with severe periodontitis. Among the patients who reported with asymptomatic CCs, $5.74 \%$ of the patients were diagnosed with mild periodontitis, $2.87 \%$ of the patients were diagnosed with moderate periodontitis, and $10.25 \%$ of the patients were diagnosed with severe periodontitis. Chi-square test shows that there is a significant relationship between type of CC and severity of periodontitis with a $p$ value of 0.015 (Fig. 3).

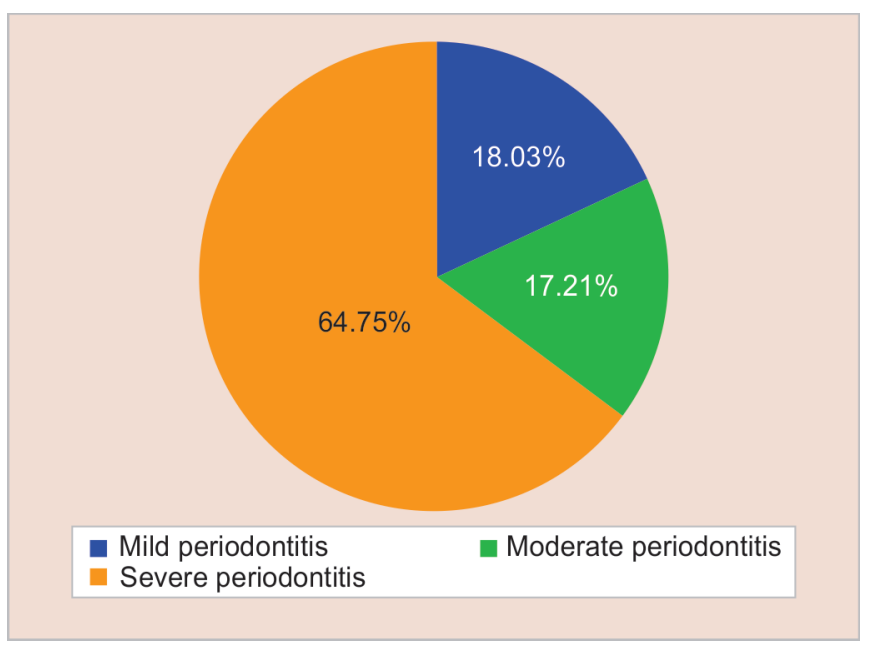

Fig. 2: Pie chart representing severity of periodontitis in the study population. Blue denotes mild periodontitis ( $L O A=<3 \mathrm{~mm}$ ), green denotes moderate periodontitis ( $L O A=3-4 \mathrm{~mm}$ at $\geq 30 \%$ sites), and orange denotes severe periodontitis (LOA $\geq 5 \mathrm{~mm}$ at $\geq 30 \%$ of sites). More than half of the study population were diagnosed with severe periodontitis $(64.75 \%)$, followed by mild periodontitis (18.03\%), and moderate periodontitis (17.21\%)

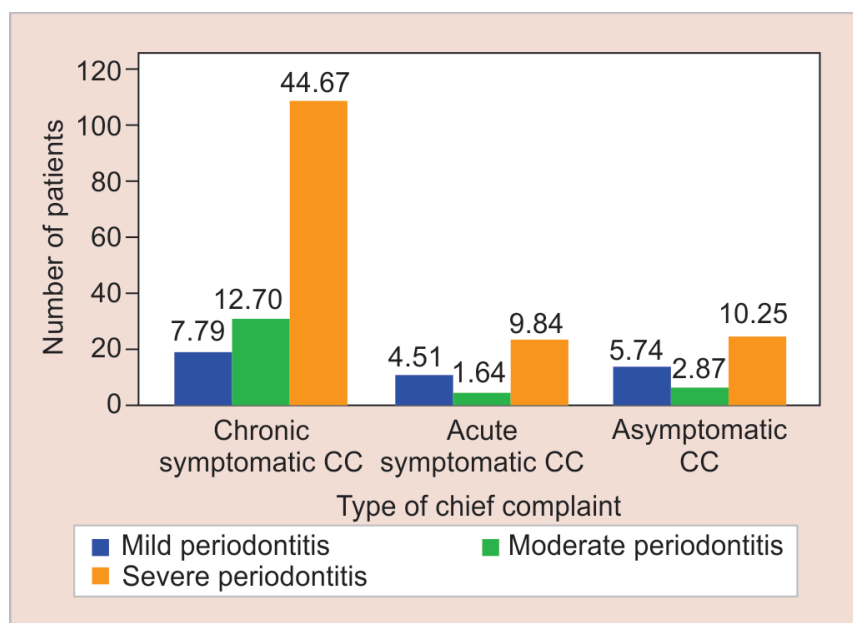

Fig. 3: Bar graph depicting the relationship between the type of chief complaints and number of patients with periodontitis. $X$-axis represents type of chief complaints and $Y$-axis represents number of patients with periodontitis included in the study. Among the patients who reported with chronic symptomatic CC, most of them were diagnosed with severe periodontitis (orange), followed by moderate (green), and mild periodontitis (blue). There is a significant relationship between type of chief complaint and severity of periodontitis (Chi-square test; $p$ value $<0.05$ )

Among patients who were diagnosed with mild periodontitis, missing teeth was the most common CC (31.82\%), followed by toothache (25\%), and swollen gums (11.36\%) (Fig. 4). Among patients who were diagnosed with moderate periodontitis, bleeding gums were the most common CC (38.1\%), followed by tooth moved position (16.67\%), and missing teeth (11.90\%) (Fig. 5). Among patients who were diagnosed with severe periodontitis, bleeding gums were the most common CC (24.68\%), followed by mobile teeth $(18.35 \%)$, followed by missing teeth (15.19\%), and toothache (15.19\%) (Fig. 6). 


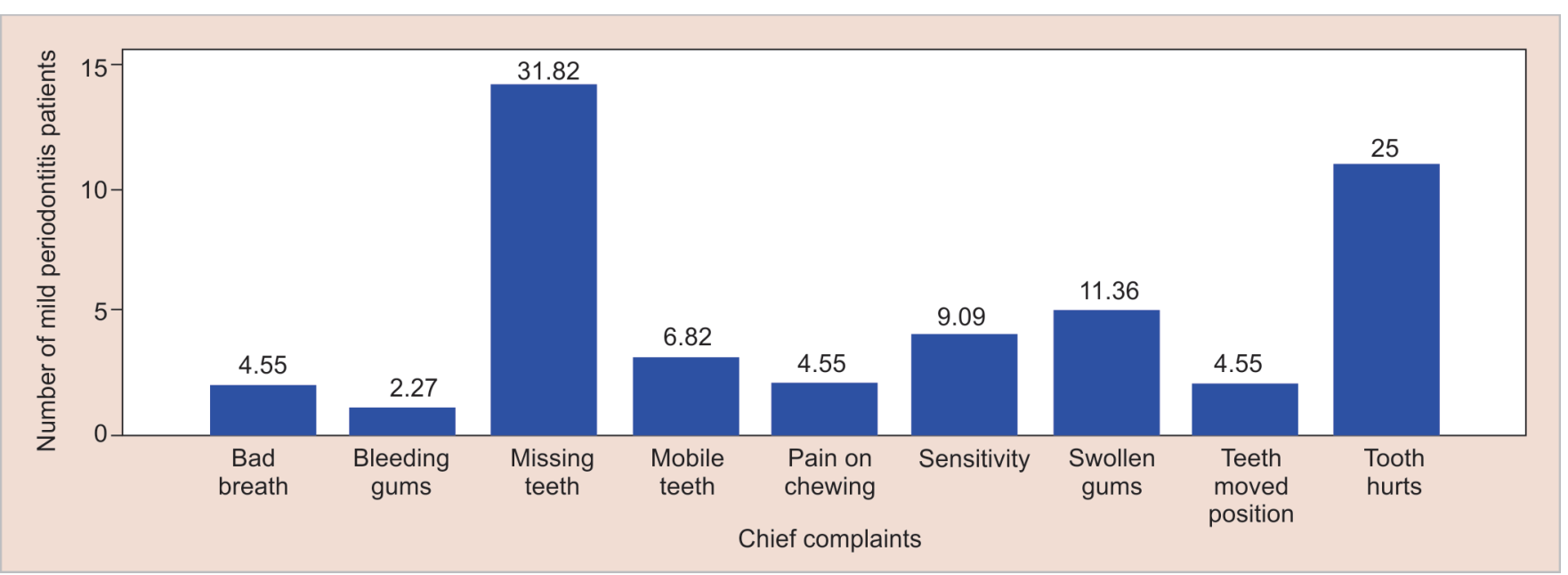

Fig. 4: Bar graph depicting the distribution of chief complaints among the patients with mild periodontitis. Blue denotes mild periodontitis $(\mathrm{LOA}=<3 \mathrm{~mm}) . X$-axis represents various chief complaints reported by the patients and $Y$-axis represents the number of patients who were diagnosed with mild periodontitis. Among patients who were diagnosed with mild periodontitis, missing teeth was the most common CC (31.82\%), followed by toothache (25\%), and swollen gums (11.36\%)

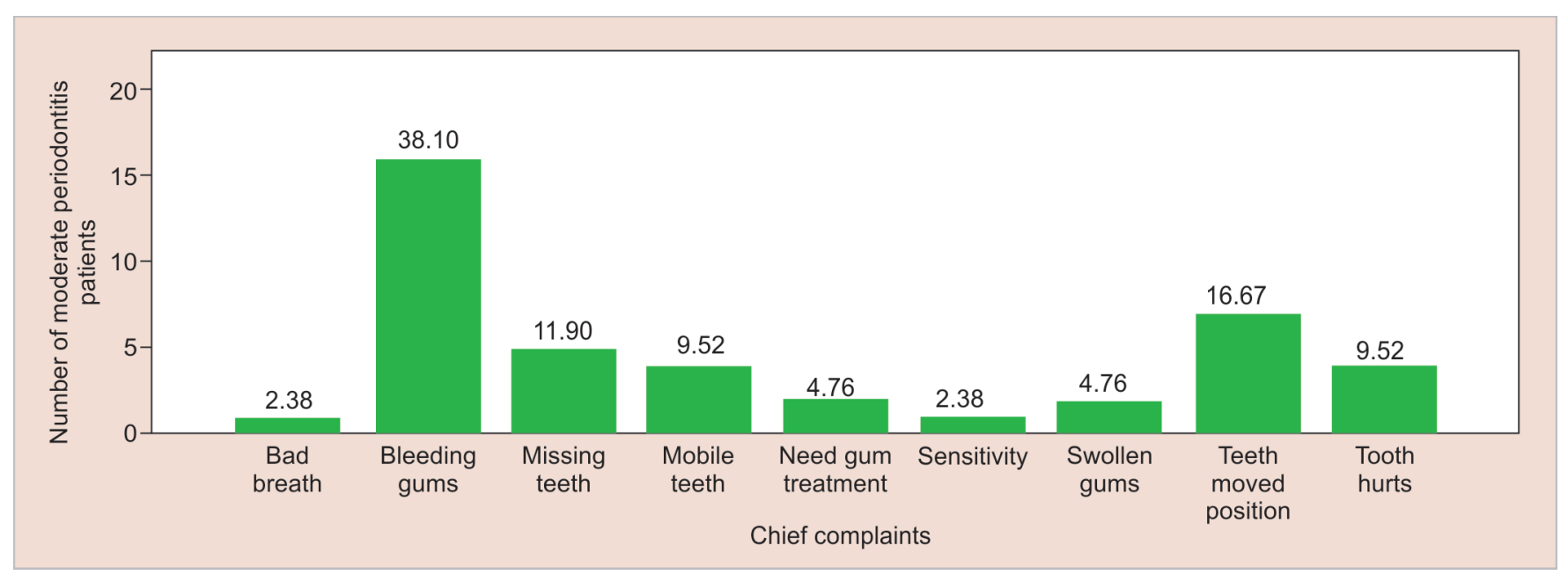

Fig. 5: Bar graph depicting the distribution of chief complaints among the patients with moderate periodontitis. Green denotes moderate periodontitis ( $\mathrm{LOA}=3-4 \mathrm{~mm}$ at $\geq 30 \%$ sites). $X$-axis represents various chief complaints reported by the patients and $Y$-axis represents the number of patients who were diagnosed with moderate periodontitis. Among patients who were diagnosed with moderate periodontitis, bleeding gums were the most common CC (38.1\%), followed by tooth moved position (16.67\%), and missing teeth (11.90\%)

\section{Discussion}

In the present study, irrespective of the type of periodontitis, the first most common CC is bleeding gums (22.95\%), second most common CC is missing teeth (17.62\%), and the third most common CC is tooth pain (15.98\%). The finding is similar to the study performed by Demetriou et al., ${ }^{26}$ and Brown et al., ${ }^{27}$ where gingival bleeding was the most common clinical symptom that directed patients to seek periodontal treatment. These findings were not in accordance with the studies by Brunsvold et al., 1999, where the most common CC was "I was told I have gum disease", which was ${ }^{19}$ reported by $31.4 \%$ of the subjects. Second most common CC reported was, "I would like to save my teeth", which was reported by $20.4 \%$ subjects. This study was performed in American population at the University of Texas. A study performed by Yeh and Lai in the Taiwan population showed that most commonly patients stated that "they need gum treatment". ${ }^{28}$ This difference in the findings is because of the lack of awareness about gum diseases among Indian population.
In the present study, only $6.56 \%$ of the subjects complained that "teeth moved position". This finding is similar to the results by Michael et al., where only $9.4 \%$ of the subjects reported a CC of pathological tooth migration. ${ }^{19}$ This finding was opposing to the results obtained by Towfighi et al., where they found abnormal tooth migration in $30 \%$ of the subjects. ${ }^{29}$ This difference suggests that some subjects with pathological tooth migration do not report it as a CC.

In the present study, $2.05 \%$ of subjects reported having bad breath. This finding is similar to results from Michael et al., where $6.8 \%$ of subjects reported bad breath as CC. ${ }^{19}$ This may be due to the subject's reluctance to discuss bad breath, which is often considered a personal matter, or they are not aware of their bad breath.

In the present study, more than half of the study population were diagnosed with moderate to severe periodontitis. This is similar to the results by Brown et al., where more than one half of the U.S. population which were studied were diagnosed with moderate to severe periodontitis. ${ }^{30}$ No previous study has been 


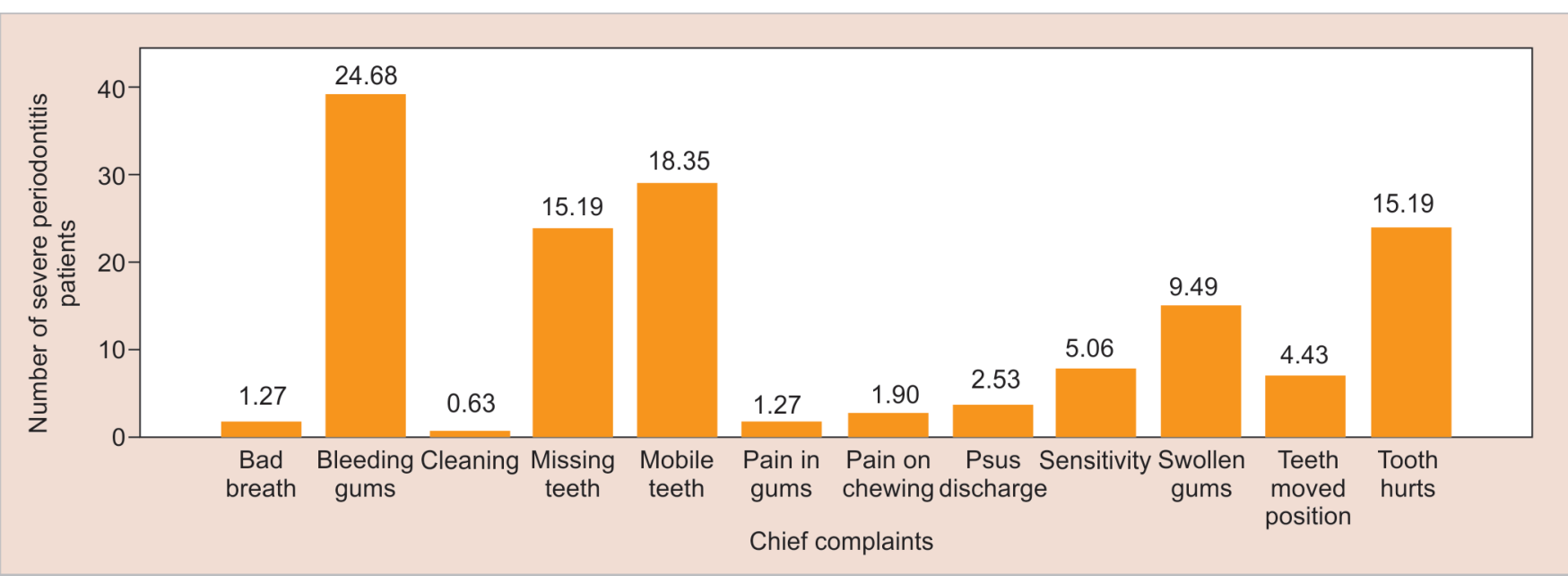

Fig. 6: Bar graph depicting the distribution of chief complaints among the patients with severe periodontitis. Orange denotes severe periodontitis (LOA $\geq 5 \mathrm{~mm}$ at $\geq 30 \%$ of sites). $X$-axis represents various chief complaints reported by the patients and $Y$-axis represents the number of patients who were diagnosed with severe periodontitis. Among patients who were diagnosed with severe periodontitis, bleeding gums were the most common CC (24.68\%), followed by mobile teeth (18.35\%), followed by missing teeth (15.19\%), and toothache $(15.19 \%)$

conducted to assess the relationship between CCs with severity of periodontitis.

\section{Limitations and Future Scope}

Our study solely depends on the ability of the patients to express their complaints. Patients may not reveal all his problems, or may even hide some of his problems, assuming that they may be irrelevant. Difference in the interpretation of the CCs by the examining dentist can affect the recording of the CC. Factors like educational level, travel distance, smoking status, and socioeconomic status may influence patient compliance, they were not considered. Further longitudinal studies with larger sample size are needed to be performed.

\section{CONCLUSION}

The CC is the initial step in dental history taking and is a brief statement explaining the symptom and condition that demands a dental visit. Within the limitations of the study, the results of this study suggest that the CC is an essential component of the dental history, with bleeding gums being the most commonly reported among the patients seeking periodontal therapy.

\section{Clinical Significance}

Patient's CC is considered as a vital component for providing competent and good quality healthcare. The severity of the disease is said to have an influence on the CCs reported by the patients. Thus, determining the relationship between the CCs and severity of periodontitis in patients seeking periodontal therapy is essential.

\section{References}

1. Priyanka S, Kaarthikeyan G, Nadathur JD, et al. Detection of cytomegalovirus, Epstein-Barr virus, and torque Teno virus in subgingival and atheromatous plaques of cardiac patients with chronic periodontitis. J Indian Soc Periodontol 2017;21(6):456-460. DOI: 10.4103/jisp.jisp_205_17.

2. Varghese SS, Thomas $\mathrm{H}$, Jayakumar ND, et al. Estimation of salivary tumor necrosis factor-alpha in chronic and aggressive periodontitis patients. Contemp Clin Dent 2015;6(Suppl 1):S152-S156. DOI: 10.4103/0976-237X.166816.

3. Mootha A, Malaiappan S, Jayakumar ND, et al. The effect of periodontitis on expression of interleukin-21: a systematic review. Int J Inflam 2016;2016:3507503. DOI: 10.1155/2016/3507503.

4. Khalid W, Varghese SS, Sankari M, et al. Comparison of Serum levels of endothelin-1 in chronic periodontitis patients before and after treatment. J Clin Diagn Res 2017;11(4):ZC78-ZC81. DOI: 10.7860/ JCDR/2017/24518.9698.

5. Khalid W, Vargheese SS, Lakshmanan R, et al. Role of endothelin-1 in periodontal diseases: A structured review. Indian J Dent Res 2016;27(3):323-333. DOI: 10.4103/0970-9290.186247.

6. Ramesh A, Varghese SS, Jayakumar ND, et al. Chronic obstructive pulmonary disease and periodontitis - unwinding their linking mechanisms. J Oral Biosci 2016;58:23-26. DOI: 10.1016/ j.job.2015.09.001.

7. Kavarthapu A, Thamaraiselvan M. Assessing the variation in course and position of inferior alveolar nerve among south indian population: a cone beam computed tomographic study. Indian J Dent Res 2018;29(4):405-409. DOI: 10.4103/ijdr.IJDR_418_17.

8. Ramesh A, Ravi S, Kaarthikeyan G. Comprehensive rehabilitation using dental implants in generalized aggressive periodontitis. J Indian Soc Periodontol 2017;21(2):160-163. DOI: 10.4103/jisp.jisp_ 213_17.

9. Ramesh A, Varghese SS, Doraiswamy JN, et al. Herbs as an antioxidant arsenal for periodontal diseases. J Intercult Ethnopharmacol 2016;5(1):92-96. DOI: 10.5455/jice.20160122065556.

10. Ramamurthy J, Mg V. Comparison of effect of Hiora mouthwash vs chlorhexidine mouthwash in gingivitis patients: a clinical trial. Asian J Pharm Clin Res 2018;11(7):84-88. DOI: 10.22159/ajpcr.2018. v11i7.24783.

11. Ramesh A, Vellayappan R, Ravi S, et al. Esthetic lip repositioning: a cosmetic approach for correction of gummy smile-A case series. J Indian Soc Periodontol 2019;23(3):290-294. DOI: 10.4103/jisp. jisp_548_18.

12. Thamaraiselvan M, Elavarasu S, Thangakumaran S, et al. Comparative clinical evaluation of coronally advanced flap with or without platelet rich fibrin membrane in the treatment of isolated gingival recession. J Indian Soc Periodontol 2015;19(1):66-71. DOI: 10.4103/0972$124 X .145790$

13. Panda S, Jayakumar ND, Sankari M, et al. Platelet rich fibrin and xenograft in treatment of intrabony defect. Contemp Clin Dent 2014;5(4):550-554. DOI: 10.4103/0976-237X.142830. 
14. Ravi S, Malaiappan $S$, Varghese $S$, et al. Additive effect of plasma rich in growth factors with Guided tissue regeneration in treatment of Intrabony defects in patients with chronic periodontitis: a splitmouth Randomized Controlled clinical trial. J Periodontol 2017;88(9): 839-845. DOI: 10.1902/jop.2017.160824.

15. Avinash K, Malaippan S, Dooraiswamy JN. Methods of isolation and characterization of stem cells from different regions of oral cavity using markers: a systematic review. Int J Stem Cells 2017;10(1):12-20. DOI: 10.15283/ijsc17010.

16. Shaju JP, Zade RM, Das M. Prevalence of periodontitis in the indian population: a literature review. J Indian Soc Periodontol 2011;15(1): 29-34. DOI: 10.4103/0972-124X.82261.

17. Baehni P, Tonetti MS. Group 1 of the European workshop on periodontology. conclusions and consensus statements on periodontal health, policy and education in Europe: a call for actionconsensus view 1. consensus report of the 1st European workshop on periodontal education. Eur J Dent Educ 2010;14(Suppl 1):2-3. DOI: 10.1111/j.1600-0579.2010.00619.x.

18. Demetriou N, Parashis A, Tsami-Pandi A. Relationship between age and clinical symptoms of periodontal disease. Stomatologia 1990;47(4):231-241. Available from: https://www.ncbi.nlm.nih.gov/ pubmed/2131686.

19. Brunsvold MA, Nair P, Oates Jr. TW. Chief complaints of patients seeking treatment for periodontitis. J Am Dent Assoc 1999;130(3): 359-364. DOI: 10.14219/jada.archive.1999.0205.

20. Gordon SM, Dionne RA. The integration of clinical research into dental therapeutics. J Am Dent Assoc 2004;135(11):1537-1542. DOI: 10.14219/ jada.archive.2004.0079.

21. Greenberg MS. Form expert panels to develop diagnosis and management protocols. Oral Surg Oral Med Oral Pathol Oral Radiol Endod 2003;95(3):265. DOI: 10.1067/moe.2003.137.
22. Al-Zahrani MI. Parent's knowledge and attitude towards their children's oral health and timing of the first dental visit in AL-Madinah city. Saudi Dent J 2019;31:S45. DOI: 10.1016/j.sdentj.2019. 02.023.

23. Bae SM, Chung WG, Jang JH, et al. Competencies for entry into the Profession of dental hygiene. J Dent Hyg Sci 2017;17(3):193-201. DOI: 10.17135/jdhs.2017.17.3.193.

24. Lewis $C$, Lynch $H$, Johnston B. Dental complaints in emergency departments: a national perspective. Ann Emerg Med 2003;42(1): 93-99. DOI: 10.1067/mem.2003.234.

25. Aljohani HA. Association between hemoglobin level and severity of chronic periodontitis. J King Abdulaziz Univ Med Sci 2010;98(277):1-24. Available from: https://www.kau.edu.sa/Files/320/ Researches/57892_28023.pdf.

26. Demetriou N, Tsami-Pandi A, Parashis A. Is it possible for periodontal patients to recognize periodontal disease. Stomatologia 1991;47(56):284-295. Available from: https://www.ncbi.nlm.nih.gov/ pubmed/1949094.

27. Brown LJ, Oliver RC, Löe H. Periodontal diseases in the U.S. in 1981 prevalence, severity, extent, and role in tooth mortality. J Periodontol 1989;60(7):363-370. DOI: 10.1902/jop.1989.60.7.363.

28. Yeh HC, Lai H. Association between patients' chief complaints and their compliance with periodontal therapy. J Clin Periodontol 2011;38(5):449-456. DOI: 10.1111/j.1600-051X.2011.01711.x.

29. Towfighi PP, Brunsvold MA, Storey AT, et al. Pathologic migration of anterior teeth in patients with moderate to severe periodontitis. J Periodontol 1997;68(10):967-972. DOI: 10.1902/jop.1997.68. 10.967.

30. Brown LJ, Löe H. Prevalence, extent, severity and progression of periodontal disease. Periodontol 2000 1993;2:57-71. DOI: 10.1111/ j.1600-0757.1993.tb00220.x. 